\title{
Applicability of the STOPPISTART criteria to older polypathological patients in a long-term care hospital
}

\author{
Julia Hernandez Martin, ${ }^{1}$ Virginia Merino-Sanjuán, ${ }^{2}$ Juan Peris-Martí, ${ }^{3}$ \\ Marta Correa-Ballester, ${ }^{1}$ Raquel Vial-Escolano, ${ }^{4}$ Matilde Merino-Sanjuán ${ }^{2}$
}

${ }^{1}$ Farmacia, Hospital San José Teruel, Spain

${ }^{2}$ Instituto Interuniversitario Reconocimiento Molecular y Desarrollo Tecnológico, Departamento de Farmacia y Tecnología Farmacéutica y Parasitología de la Universidad de Valencia., Valencia, Spain ${ }^{3}$ Servicio de Farmacia, Residencia Personas Mayores Dependientes La Cañada.,

Valencia, Spain

${ }^{4}$ Unidad de Geriatría, Hospital San José., Teruel, Spain

\section{Correspondence to}

Dr Julia Hernandez Martin, Farmacia, Hospital San José, Teruel, 44001, Spain; jhernandezm@salud.aragon.es

Received 13 March 2017 Revised 9 May 2017

Accepted 6 July 2017 Published Online First 9 August 2017

EAHP Statement 4: Clinical Pharmacy Services.

\begin{abstract}
Objectives To analyse the applicability of the STOPP/

START criteria as a tool to identify patients with potentially inappropriate medications (PIM) during pharmaceutical validation of prescriptions in a longterm care hospital, to identify risk factors for PIM and to characterise the physiological systems and drugs more frequently associated with these PIM.

Methods An interventional, prospective and longitudinal study was conducted in polypathological patients aged $>65$ years. Usual pharmaceutical care and the STOPPISTART criteria were used to identify PIM and to plan pharmaceutical interventions at admission. At discharge, the discharge summaries were reviewed using the STOPP/START criteria.
\end{abstract}

Results 112 patients were included. The prevalence of patients with PIM at admission was $76.8 \%$. The STOPP criteria identified a high number of PIM and almost all entailed pharmaceutical intervention. On the other hand, most of the START criteria identified did not entail pharmaceutical intervention. Usual pharmaceutical care detected a different type of PIM; a high percentage of pharmaceutical interventions to resolve them were accepted. At discharge, the prevalence of patients with PIM was $61.3 \%$. At admission, none of the analysed variables was associated with the PIM identified using any of the tools. At discharge STOPP criteria identified a higher percentage of patients with PIM in the geriatric outpatient consultation group.

Conclusions The prevalence of PIM in older polypathological patients is high. The STOPP criteria are useful for reducing inappropriate prescribing during the pharmaceutical validation process. In contrast, routine incorporation of the START criteria in the pharmaceutical validation may be not necessary in a hospital of this type.

\section{INTRODUCTION}

Use of drugs in the elderly can be considered a health problem ${ }^{12}$ since the prevalence of potentially inappropriate medications (PIM) in people aged $>65$ years in primary healthcare is $20.5 \%$, up to $40 \%$ for nursing home residents in the USA, ${ }^{3}$ and 95.1\% for hospitalised older adults in Spain. ${ }^{4}$

Polypharmacy and pluripathology are factors associated with PIM, as well as pharmacokinetic and pharmacodynamic change, which are common in older people. ${ }^{35}$ In other studies, ${ }^{6-8}$ place of residence, female sex and depression were shown to be other factors associated with PIM.

Several tools are available for assessing the appropriateness of drug treatment and for reducing use of PIM in the elderly; these are divided into implicit and explicit methods. The explicit methods include
Beers criteria, IPET (Improved Prescribing in the Elderly Tool) and the STOPP/START criteria (Screening Tool of Older Person's Prescriptions/ Screening Tool to Alert doctors to Right Treatment). ${ }^{9}$ STOPP/START consists of 65 indicators of potentially inadequate treatments, including drugdrug and drug-disease interactions (STOPP), and 22 indicators of prescribing omissions on the basis of an exact diagnosis if there is no contraindication (START).

Pharmacist intervention has been shown to reduce significantly the number of PIM and to improve prescriptions for fragile elderly patients at hospital admission. ${ }^{2}$ In addition, several studies have shown that the inclusion of a pharmacist in the healthcare team responsible for elderly patients reduces the negative outcomes associated with medication. ${ }^{10}$

Few studies have been carried out in long-term care hospitals that use the STOPP/START criteria and currently, no tool is used to improve medication appropriateness. Therefore, this study aimed to analyse the applicability of the STOPP/ START criteria as a tool to identify patients with PIM to improve the quality of pharmacotherapeutical practice.

\section{METHODS}

\section{Study setting and population}

A 6-month interventional, prospective and longitudinal study was conducted between March and August 2014.

The study was carried out at a long-term care hospital with 48 beds. All patients admitted to the hospital during the study period who met the following criteria were included: aged $>65$ years and polypathological. According to the Andalusian Public Health System classification ${ }^{11}$ polypathological patients were considered to be those who had chronic diseases included in two or more of the clinical categories shown in box 1. Patients had to give their informed written consent for inclusion in the study.

Patients who were already included in the study during the recruitment period and were readmitted later and those admitted to the palliative care service were excluded.

\section{Data collection}

The following data were collected: sociodemographic variables (age, gender, admission service and origin); functional variables (Barthel index and Pfeiffer index); clinical variables (number of chronic diseases at admission); prognosis variables (Charlson index, Profund index and simplified 


\section{Box 1 Definition of a polypathological patient ${ }^{11}$}

\section{Category A}

- A.1 Chronic heart failure with past/present stage II dyspnoea according to NYHA*

- A.2 Coronary heart disease

Category B

- B.1 Vasculitides and/or systemic autoimmune diseases

- B.2 Chronic renal disease (creatininaemia N1.4/1.3 mg/dL in men/women or proteinuria ${ }^{\dagger}$, for $\geq 3$ months)

\section{Category C}

- Chronic lung disease with past/present stage 2 dyspnoea according to the MRC ${ }^{\ddagger}$, or FEV1 $<65 \%$, or basal Sat02 $\leq 90 \%$

Category D

- D.1 Chronic inflammatory bowel disease

- D.2 Chronic liver disease with evidence of portal hypertension ${ }^{\S}$

Category E

- E.1 Stroke

- E.2 Neurological disease with permanent motor deficit, leading to severe impairment of basic activities of daily living (Barthel index $<60$ ).

- E.3 Neurological disease with permanent moderate-severe cognitive impairment (Pfeiffer's test with $\geq 5$ errors).

\section{Category $\mathrm{F}$}

- F.1 Symptomatic peripheral artery disease

- F.2 Diabetes mellitus with proliferative retinopathy or symptomatic neuropathy

\section{Category G}

- G.1 Chronic anaemia ( $\mathrm{Hb}<10 \mathrm{~g} / \mathrm{dL}$ for $\geq 3$ months) due to digestive-tract losses or acquired haemopathy not a side effect of treatment

- G.2 Solid-organ or haematologically active neoplasia not a tributary of treatment with curative intention.

\section{Category $\mathrm{H}$}

- Chronic osteoarticular disease, leading to severe impairment of basic activities of daily living (Barthel index $<60$ )

*Slight limitation of physical activity. Comfortable at rest, but ordinary physical activity results in fatigue, palpitation or dyspnoea.

†Albumin/creatinine index $>300 \mathrm{mg} / \mathrm{g}$, microalbuminuria $>3 \mathrm{mg} /$ $\mathrm{dL}$ in urine, albumin $>300 \mathrm{mg} /$ day in 24-hour urine, or albuminuria/ $\min >200 \mu \mathrm{g} / \mathrm{min}$.

¥Short of breath when hurrying or walking up a slight hill.

§Presence of clinical, analytical, echographic or endoscopic data of portal hypertension.

FEV1, forced expiratory volume in one second; MRC, Medical Research Council; NYHA, New York Heart Association; Sat02, oxygen saturation.

Profunction index) and pharmacotherapy variables (number of drugs at the time of the first review and number of drugs prescribed in the hospital discharge report). To count the number of drugs prescribed, 'as needed' treatments and intravenous fluid therapy were included but enteral nutrition or nutritional supplements products were not.

Clinical pharmacists combined their usual pharmaceutical care without tools (consultation of product data sheet, medication administration guides, usual databases used in the hospital and lists of low therapeutic value drugs) and the STOPP/START criteria to perform a comprehensive medication review in all patients included. The pharmacist informed the physician of all detected PIM with the exception of the PIM identified by the START criteria in patients with significant functional impairment or high or intermediate risk of death in 1 year.

Discharge summaries were reviewed using the STOPP/START criteria. Pharmaceutical interventions were not made to resolve the detected PIM since the review was conducted the day after hospital discharge and the PIM detected did not affect patient safety.

\section{Data analysis}

Data were analysed using SPSS 22.0. A descriptive analysis of sociodemographic, functional, clinical and prognostic variables was conducted. The Kolmogorov-Smirnov test was used to determine the normal distribution of the quantitative variables.

The prevalence of patients with PIM at admission was calculated as the proportion of all patients included in the study, whereas at hospital discharge the prevalence was calculated from the patients who completed the study.

A non-parametric McNemar test was used to compare the prevalence of patients with PIM identified by STOPP/START criteria combined or STOPP or START separately, between admission and discharge.

Univariate analyses were performed to identify factors associated with PIM identified by STOPP/START criteria combined or STOPP or START separately. A X ${ }^{2}$ association test (or Fisher's exact test if the expected frequency was $<20 \%$ ) was used for the analysis.

$\mathrm{p}<0.05$ was considered statistically significant.

The degree of applicability of the STOPP/START criteria was assessed through indicators $\mathrm{I}_{1}, \mathrm{I}_{2}$ and $\mathrm{I}_{3}$ :

$\mathrm{I}_{1}$ : number and percentage of PIM identified with STOPP criteria, with START criteria and with the usual care divided by the total number of PIM identified.

$\mathrm{I}_{2}$ : number and percentage of PIM identified with STOPP criteria, START criteria and with the usual care that entailed pharmaceutical interventions divided by the total number of PIM identified with each method.

$\mathrm{I}_{3}$ : number and percentage of PIM identified with each method that were modified after the pharmaceutical intervention divided by the total number of PIM identified with each method that entailed pharmaceutical intervention.

\section{Ethical considerations}

The study protocol was approved by the institutional review board (clinical research ethics committee of Aragon; CEICA), and informed written consent was obtained from all participants.

\section{RESULTS}

\section{Study population}

One hundred and twelve patients met the inclusion criteria and all agreed to participate in the study. Only 106 patients completed the study: five patients died at admission and one patient was transferred urgently to the reference acute care hospital.

Sociodemographic, functional, clinical, prognostic and pharmacotherapy characteristics of the patients included in the study are presented in table 1.

\section{Potentially inappropriate medication}

The prevalence of patients with PIM at admission was 76.8\% $(\mathrm{n}=86)$. The STOPP/START criteria identified PIM in $66.1 \%$ of the patients $(\mathrm{n}=74)$, the STOPP criteria in $29.5 \%$ of patients 


\begin{tabular}{|c|c|}
\hline \multicolumn{2}{|l|}{ Age (years) } \\
\hline Mean \pm SD & $85.9 \pm 6.8$ \\
\hline Range & $66-99$ \\
\hline \multicolumn{2}{|l|}{ Interval (years), n (\%) } \\
\hline $66-80$ & $20(17.9 \%)$ \\
\hline$\geq 81$ & $92(82.1 \%)$ \\
\hline Female gender, $\mathrm{n}(\%)$ & $56(50 \%)$ \\
\hline \multicolumn{2}{|l|}{ Admission Service Unit in the long-term care hospital, $\mathrm{n}(\%)$} \\
\hline Acute care & $56(50 \%)$ \\
\hline Rehabilitation & $36(32.1 \%)$ \\
\hline Convalescence & $20(17.9 \%)$ \\
\hline \multicolumn{2}{|l|}{ Origin, $n(\%)$} \\
\hline Unit of the acute care hospital & $52(46.4 \%)$ \\
\hline Emergency department of the acute care hospital & $54(48.2 \%)$ \\
\hline Geriatric outpatient consultation & $6(5.4 \%)$ \\
\hline \multicolumn{2}{|l|}{ Degree of dependence according to Barthel index, $\mathrm{n}(\%)$} \\
\hline Total (<20 points) & $53(47.3 \%)$ \\
\hline Severe (20-35 points) & $29(25.9 \%)$ \\
\hline Moderate (40-55 points) & $17(15.2 \%)$ \\
\hline Slight (60-85 points) & $8(7.1 \%)$ \\
\hline Independent (90-100 points) & $5(4.5 \%)$ \\
\hline \multicolumn{2}{|c|}{$\begin{array}{l}\text { Degree of cognitive impairment according to the Pfeiffer index, } \\
\mathrm{n}(\%)\end{array}$} \\
\hline Severe (8-10 errors) & $42(37.5 \%)$ \\
\hline Moderate (5-7 errors) & $9(8.0 \%)$ \\
\hline Mild (3-4 errors) & $23(20.5 \%)$ \\
\hline Normal cognitive function ( $0-2$ errors) & $38(33.9 \%)$ \\
\hline \multicolumn{2}{|l|}{ Number of pathologies } \\
\hline Mean, range & $3,2-7$ \\
\hline \multicolumn{2}{|l|}{ Comorbidity, n (\%) } \\
\hline Charlson index $\leq 6$ & $24(21.4 \%)$ \\
\hline Charlson index 7-10 & $77(68.8 \%)$ \\
\hline Charlson index $\geq 11$ & $11(9.8 \%)$ \\
\hline \multicolumn{2}{|c|}{ Risk of death in the next year according to Profund index, $\mathrm{n}(\%)$} \\
\hline High ( $\geq 11$ points) & $57(50.9 \%)$ \\
\hline Intermediate-high (7-10 points) & $27(24.1 \%)$ \\
\hline Low-intermediate (3-6 points) & $22(19.6 \%)$ \\
\hline Low (0-2 points) & $6(5.4 \%)$ \\
\hline \multicolumn{2}{|c|}{$\begin{array}{l}\text { Risk of functional decline in the following year according to } \\
\text { simplified Profunction index, } \mathrm{n}(\%)\end{array}$} \\
\hline High ( $\geq 4$ points) & $37(33.0 \%)$ \\
\hline Intermediate-high (three points) & $29(25.9 \%)$ \\
\hline Low-intermediate (1-2 points) & $42(37.5 \%)$ \\
\hline Low (0 points) & $4(3.6 \%)$ \\
\hline \multicolumn{2}{|l|}{ Medication prescribed at admission } \\
\hline $\begin{array}{l}\text { Mean } \pm S D \\
\text { Range }\end{array}$ & $\begin{array}{l}11.5 \pm 3.1 \\
4-21\end{array}$ \\
\hline$\leq 9$ Drugs $n(\%)$ & $25(22.3 \%)$ \\
\hline$\geq 10$ Drugs $\mathrm{n}(\%)$ & $87(77.7 \%)$ \\
\hline \multicolumn{2}{|l|}{ Medication prescribed at discharge } \\
\hline Mean \pm SD & $10.0 \pm 3.4$ \\
\hline Range & $3-21$ \\
\hline$\leq 9$ Drugs $\mathrm{n}(\%)$ & $49(46.2 \%)$ \\
\hline$\geq 10$ Drugs $\mathrm{n}(\%)$ & $57(53.8 \%)$ \\
\hline
\end{tabular}

$(n=33)$, the START criteria in $56.3 \%$ of patients $(n=63)$ and the usual pharmaceutical care in $26.8 \%$ of patients $(n=30)$.

The prevalence of patients with PIM identified during discharge summaries review was $61.3 \%(n=65)$. The STOPP criteria detected PIM in $20.8 \%$ of patients $(n=22)$ and the START criteria in $50.9 \%$ of patients $(n=54)$. Although these percentages are lower than the admission values, the differences do not reach statistical significance.

Details of the most common drugs involved in PIM are shown in table 2 .

For the STOPP criterion 'Any duplicate drug class prescription', has been posted the two drugs involved.

Tables 3 and 4 show the frequency of PIM according to the STOPP and START criteria, at admission and at discharge, respectively.

\section{Pharmaceutical interventions}

A total of 87 pharmaceutical interventions were made. The main types of pharmaceutical interventions were: to change a medication $(28.7 \%)$, to discontinue a treatment $(27.6 \%)$, to start a medication (18.4\%) and to change the dose or the dosing interval (17.2\%). Of these 87 pharmaceutical interventions, 65 were accepted (74.7\%).

Applicability of STOPP/START criteria. Results of the indicators The degree of applicability of the STOPP/START criteria evaluated from $\mathrm{I}_{1}, \mathrm{I}_{2}, \mathrm{I}_{3}$ indicators is shown in figure 1 .

\section{Factors associated with PIM}

At hospital admission

Sociodemographic variables (gender, age, admitting service and origin) were not statistically associated with the identified PIM, irrespective of the tools used. Neither were the number of medications, degree of comorbidity or prognostic variables (risk of death and risk of functional decline in the following year). Differences were found between the prevalence of PIM identified by STOPP criteria in patients receiving $>10$ drugs and the group receiving $\leq 9(33.3 \%$ vs $16 \%)$, although these were not statistically significant. The PIM detected were considerably higher in women than in men using the START criteria (64.3\% vs $48.2 \%)$, but the differences were not statistically significant.

\section{At hospital discharge}

STOPP criteria identified a higher percentage of patients with PIM in the group from the geriatric outpatient consultation $(66.7 \%)$ than in patients from a unit of the acute care hospital $(18.4 \%)$ or from emergency department of the acute care hospital (17.6\%). However, the sample comprised only six patients.

The other variables showed no statistical significance, although it should be noted that START criteria identified a slight greater prevalence of patients with PIM in groups with high or intermediate-high risk of death or functional decline in the following year than in other groups.

\section{DISCUSSION}

\section{Potentially inappropriate medication}

The use of PIM in elderly patients is the cause of $6-30 \%$ of hospital admissions. ${ }^{12}$ Moreover, PIM are also common during the stay in hospital and at discharge, ${ }^{413}$ as was found in this study.

PIM were identified in $66.1 \%$ of the patients by the STOPP/ START criteria and in $28.1 \%$ by usual pharmaceutical care. These results are in line with other studies, ${ }^{413}$ and emphasise the usefulness of the STOPP/START crieria used in conjunction with the medication review process. The global prevalence of PIM in studies that combine various tools (Beers criteria, anticholinergic risk scale, Priscus criteria) ${ }^{1415}$ is higher than with one tool alone. 
Table 2 Number and percentage of potentially inappropriate medications (PIM) identified with the methods used at hospital admission and at discharge.

\begin{tabular}{|c|c|c|c|c|c|c|}
\hline \multirow[b]{3}{*}{ Drug } & \multicolumn{6}{|l|}{ PIM, n (\%) } \\
\hline & \multicolumn{2}{|c|}{ STOPP } & \multicolumn{2}{|c|}{ START } & \multirow{2}{*}{$\begin{array}{r}\text { Usual care } \\
\text { Admission }\end{array}$} & \multirow[b]{2}{*}{ Total } \\
\hline & Admission & Discharge & Admission & Discharge & & \\
\hline Acetylsalicylic & $3(6.5 \%)$ & 0 & $21(21.9 \%)$ & $20(23.3 \%)$ & 0 & 44 (15.1\%) \\
\hline Amlodipine & $4(8.7 \%)$ & $3(10.3 \%)$ & 0 & 0 & 0 & $7(2.4 \%)$ \\
\hline Calcium+vitamin D & 0 & 0 & $14(14.6 \%)$ & $9(10.5 \%)$ & 0 & $23(7.9 \%)$ \\
\hline Ipratropium & $4(8.7 \%)$ & $1(3.5 \%)$ & 0 & 0 & 0 & $5(1.7 \%)$ \\
\hline Levofloxacin & 0 & 0 & 0 & 0 & $5(14.7 \%)$ & $5(1.7 \%)$ \\
\hline Lorazepam & $1(2.2 \%)$ & $5(17.2 \%)$ & 0 & 0 & 0 & $6(2.1 \%)$ \\
\hline Metformin & 0 & 0 & $11(11.5 \%)$ & $11(12.8 \%)$ & 0 & $22(7.6 \%)$ \\
\hline Omeprazole & 0 & 0 & 0 & 0 & $7(20.6 \%)$ & $7(2.4 \%)$ \\
\hline Quetiapine & $2(4.4 \%)$ & $5(17.2 \%)$ & 0 & 0 & 0 & $7(2.4 \%)$ \\
\hline
\end{tabular}

Table 3 Potentially inappropriate medication at admission and at discharge identified by STOPP criteria categorised by physiological system

\begin{tabular}{|c|c|c|}
\hline & \multicolumn{2}{|l|}{ PIM, n(\%) } \\
\hline & Admission & Discharge \\
\hline A. Cardiovascular system & $9(22.5 \%)$ & $4(14.8 \%)$ \\
\hline Digoxin at a long-term dose $>125 \mu \mathrm{g} /$ day with impaired renal function & 1 & 0 \\
\hline Thiazide diuretic agent with a history of gout & 1 & 1 \\
\hline$\beta$-Blocker in combination with verapamil & 1 & 0 \\
\hline Calcium channel blockers with chronic constipation & 2 & 3 \\
\hline Aspirin at dose $>150 \mathrm{mg} / \mathrm{day}$ & 3 & 0 \\
\hline Warfarin for first, uncomplicated deep venous thrombosis longer than 6 months' duration & 1 & 0 \\
\hline B. Central nervous system and psychotropic drugs & $4(10.0 \%)$ & $5(18.5 \%)$ \\
\hline Tricyclic antidepressants (TCAs) with constipation & 0 & 1 \\
\hline TCAs with an opiate or calcium channel blocker & 2 & 1 \\
\hline $\begin{array}{l}\text { Long-term (ie, }>1 \text { month), longacting benzodiazepines-eg, chlordiazepoxide, fluazepam, nitrazepam, chlorazepate } \\
\text { and benzodiazepines with longacting metabolites, e.g. diazepam }\end{array}$ & 2 & 0 \\
\hline Long-term neuroleptic agents ( $>1$ month) in those with parkinsonism & 0 & 3 \\
\hline C. Gastrointestinal system & $1(2.5 \%)$ & 0 \\
\hline Prochlorperazine (Stemetil) or metoclopramide with parkinsonism & 1 & 0 \\
\hline D. Respiratory system & $4(10.0 \%)$ & $1(3.7 \%)$ \\
\hline Nebulised ipratropium with glaucoma & 4 & 1 \\
\hline E. Musculoskeletal system & $5(12.5 \%)$ & 0 \\
\hline NSAID with moderate-to-severe hypertension & 2 & 0 \\
\hline NSAID with heart failure & 1 & 0 \\
\hline Warfarin and NSAID together & 1 & 0 \\
\hline NSAID with chronic renal failure & 1 & 0 \\
\hline F. Urogenital system & $8(20.0 \%)$ & $8(28.6 \%)$ \\
\hline$\alpha$-Blockers in men with frequent incontinence- - ie, one or more episodes of incontinence daily & 6 & 4 \\
\hline$\alpha$-Blockers with long-term urinary catheter in situ-ie, $>2$ months & 2 & 4 \\
\hline H. Drugs that adversely affect fallers & $3(7.5 \%)$ & $9(32.1 \%)$ \\
\hline Benzodiazepines & 1 & 6 \\
\hline Neuroleptic drugs & 2 & 2 \\
\hline Long-term opiates in those with recurrent falls & 0 & 1 \\
\hline J. Duplicate drug classes & $6(15.0 \%)$ & $1(3.7 \%)$ \\
\hline Any duplicate drug class prescription—eg, two concurrent opiates, NSAIDs, SSRIs, loop diuretics, ACE inhibitors & 6 & 1 \\
\hline
\end{tabular}


Table 4 Potentially Inappropriate medication at admission and at discharge identified by START criteria classified by physiological system

\begin{tabular}{|c|c|c|}
\hline & \multicolumn{2}{|l|}{ PIM, n(\%) } \\
\hline & Admission & Discharge \\
\hline A. Cardiovascular system & $27(28.1 \%)$ & $21(24.4 \%)$ \\
\hline Aspirin in the presence of chronic atrial fibrillation, where warfarin is contraindicated, but not aspirin & 0 & 2 \\
\hline $\begin{array}{l}\text { Aspirin or clopidogrel with a documented history of atherosclerotic coronary, cerebral or peripheral vascular disease in } \\
\text { patients with sinus rhythm }\end{array}$ & 7 & 4 \\
\hline ACE inhibitor with chronic heart failure & 9 & 7 \\
\hline ACE inhibitor following acute myocardial infarction & 5 & 5 \\
\hline B. Respiratory system & $1(1.0 \%)$ & $1(1.2 \%)$ \\
\hline Regular inhaled corticosteroid for moderate/severe asthma or COPD, where predicted FEV1<50\% & 1 & 1 \\
\hline E. Musculoskeletal system & $14(14.6 \%)$ & $11(12.8 \%)$ \\
\hline F. Endocrine system & $54(56.3 \%)$ & $53(61.6 \%)$ \\
\hline Metformin with type 2 diabetes \pm metabolic syndrome (in the absence of renal impairment) & 11 & 11 \\
\hline $\begin{array}{l}\text { ACE inhibitor or angiotensin receptor blocker in diabetes with nephropathy-ie, overt urinalysis proteinuria or } \\
\text { microalbuminuria ( }>30 \mathrm{mg} / 24 \text { hours }) \pm \text { serum biochemical renal impairment }\end{array}$ & 4 & 3 \\
\hline $\begin{array}{l}\text { Antiplatelet therapy in diabetes mellitus with coexisting major cardiovascular risk factors (hypertension, } \\
\text { hypercholesterolaemia, smoking history) }\end{array}$ & 14 & 14 \\
\hline Statin therapy in diabetes mellitus if coexisting major cardiovascular risk factors present & 25 & 25 \\
\hline
\end{tabular}

The prevalence of patients with PIM identified by the STOPP criteria was similar to that found by several studies reviewed. ${ }^{1617}$ However, the prevalence identified in some studies using these same criteria, was higher. ${ }^{18} 19$ These differences may be due to the origin of the patients. The patients included in our study include a high percentage of non-acute patients who had been evaluated previously by the comprehensive geriatric assessment unit, so it is possible that treatment modifications had already been made before hospital admission, whereas other studies were conducted mainly in units with acute patients. ${ }^{18} 19$

Figure 1. Results of $11,12,13$ indicators

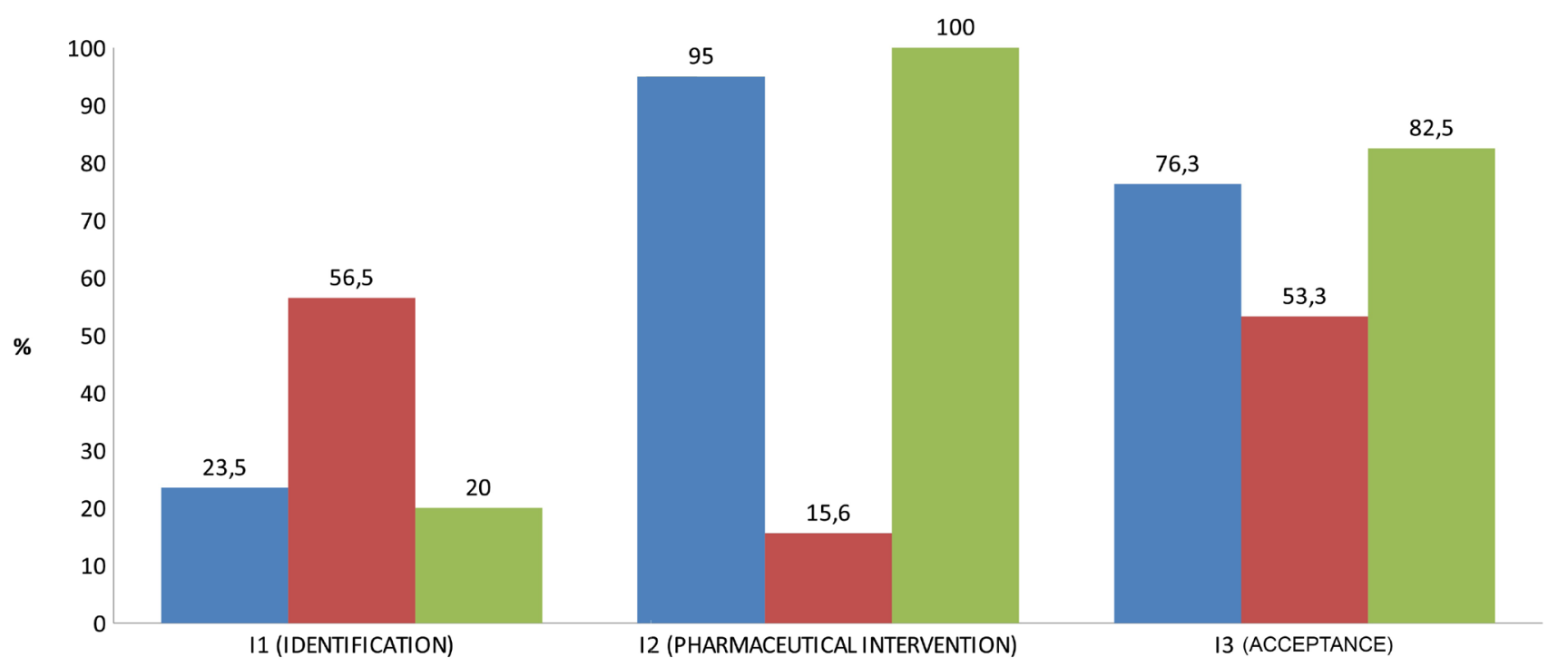

ITOPP $\square$ START $\square$ USUAL CARE

Figure 1 Results of $I_{1}, I_{2}, I_{3}$ indicators. STOPP/START, Screening Tool of Older Persons' potentially inappropriate Prescriptions/Screening Tool to Alert doctors to Right Treatment. 
In our study, strict application of the START criteria identified $56.3 \%$ of patients with PIM, which is a higher prevalence than found in other studies, ${ }^{16}$ probably because the clinical and functional status of the patients was taken into account for the application of the criteria and therefore, these START criteria were not considered to be PIM.

At hospital admission, the main physiological systems associated with the PIM identified by the STOPP criteria were the cardiovascular system (amlodipine)—also featured in several studies-,${ }^{4} 20$ the urogenital system (tamsulosin) and duplicate drug classes-also important in the majority of the studies reviewed-. ${ }^{41718}$ However, at hospital discharge, the group 'drugs that adversely affect fallers' (quetiapine and lorazepam) greatly increases, mainly owing to prescription of benzodiazepines. These data show the influence of the level of care on the need to use certain medications. This must be taken into account at discharge from hospital so that treatment changes are considered and not made automatically. The existence of a post-hospital syndrome ${ }^{21}$ is a reality that affects the fragile patient and requires treatment to be adapted to the personal situation of a patient.

In many studies 'central nervous system and psychotropic drugs' is the main physiological system involved in the PIM identified at admission, ${ }^{4-19}$ highlighting the use of longacting benzodiazepines. However, in our study, this criterion (longacting benzodiazepines) was identified in only two patients at admission, and in no patients at discharge, because the pharmaceutical interventions carried out were accepted and the prescriptions were modified.

The main physiological systems affected by applying the START criteria, both at admission and at discharge, were the endocrine system and the cardiovascular system, by omission of antiplatelet therapy and statins in patients with diabetes mellitus with cardiovascular risk factors. Neither of these criteria were met at admission, but because they were not modified during the stay, remained at discharge. This result is in agreement with other studies reviewed. ${ }^{41620}$ The START criteria 'antiplatelet therapy in diabetes mellitus' and 'statins therapy in diabetes mellitus' have been removed from the new version of the criteria owing to lack of evidence. ${ }^{22}$

The drugs mainly involved in PIM identified by usual pharmaceutical care were omeprazole and levofloxacin. These PIM could be defined as more technical, not related to explicit criteria, since they are based on specific characteristics of a patient, such as dysphagia (change from omeprazole to lansoprazole bucodispersible), the presence of a particular interaction (omeprazole-clopidogrel) or the need to individualise the dosage according to kidney function (antibiotics).

\section{Pharmaceutical interventions and applicability of STOPP/ START criteria}

The acceptance of pharmaceutical interventions, $74.7 \%$, is comparable to that seen in other studies reviewed. ${ }^{4}$

Almost all STOPP criteria identified entailed pharmaceutical intervention. The degree of acceptance of these pharmaceutical recommendations was high $(76.3 \%)$. On the other hand, most of the START criteria identified did not entail pharmaceutical intervention (84.4\%), since the patients had limited life expectancy. To start a new treatment in accordance with the START criteria it is important to take into account life expectancy of the patient or time required to obtain some benefit from treatment. ${ }^{23}$

Usual care detects a different type of PIM. Pharmaceutical interventions suggested to resolve these incidents were accepted in a high percentage $(82.5 \%)$ of cases, because in general, addition or discontinuation of medications was not required. These recommendations are intended to lead to adaptation of treatment-for example, to facilitate adhesion or to adjust drug dosing in patients with kidney disease-so acceptance by a physician does not require a complex evaluation.

\section{Factors associated with PIM}

Comparisons between studies are difficult because the level of care is not the same as in this study. None of them has been performed in a long-term care hospital and therefore patients included had a different clinical status and the age groups were different.

The analysis performed shows that gender is a variable to take into account, since the probability of PIM is higher in women. However, this variable does not reach statistical significance in our study, unlike some other studies. ${ }^{8} 18$

Age was not correlated with a higher prevalence of PIM, though statistical significance was found in other studies. ${ }^{18} 24$

The Charlson index was not identified as a risk factor for PIM with any of the tools. In contrast, other studies showed that greater comorbidity increases prescribing omissions. ${ }^{18}$

In the group of patients from outpatient geriatric consultation, STOPP criteria identified a statistically significant higher percentage with PIM at discharge, though the number of subjects in this group was small (only six), so this result can be considered irrelevant.

A higher percentage of patients with PIM was identified using the STOPP criteria in the group with $\geq 10$ medications at admission, but the differences did not reach statistical significance. However, several published studies have found a significant correlation between polypharmacy and a greater number of PIM. ${ }^{78181924}$ More START criteria were identified in the groups of patients with a limited life expectancy and more functional impairment. This was found particularly in patients with osteoporosis without calcium and vitamin $\mathrm{D}$ supplements and patients with diabetes without antiplatelet therapy. Here it is sensible not to start preventive treatments if the possible benefit might not occur.

\section{Limitations}

First, it is difficult to find factors associated with PIM owing to the small sample size. However, few studies have been carried out in long-term care hospitals so our study may be considered representative.

Second, the severity of the PIM was not determined or analysed. Also, clinical outcomes in patients were not analysed.

Finally, the lack of application of usual pharmaceutical care at hospital discharge might have led to underestimation of the percentage of patients with PIM. However, probably, the number of PIM not identified by the STOPP/START criteria at this time is small, since usual pharmaceutical care is used up to discharge day and acceptance of pharmaceutical interventions to resolve the PIM identified is high.

\section{CONCLUSION}

According to the results obtained, the STOPP criteria are useful to identify inappropriate prescribing during the pharmaceutical validation of prescription, and complement traditional pharmaceutical care. In contrast, routine incorporation of the START criteria in the pharmaceutical validation process may be not necessary in a hospital of this type because most of patients are very old, with considerable multimorbidity and limited life expectancy so the use of underprescribing tools could be controversial. 


\section{What this paper adds}

What is already known on this subject?

- The presence of potentially inappropriate medications (PIM) in hospitalised old patients is high.

- The STOPPISTART criteria help to reduce PIM in the elderly.

What this study adds?

- The STOPP criteria are useful to identify PIM during pharmaceutical validation in a long-term care hospital and complement traditional pharmaceutical care.

- The START criteria may be not necessary in elderly patients with considerable multimorbidity and limited life expectancy.

Further studies to evaluate clinical outcomes and to find factors associated with the PIM should be considered.

Contributors JHM: data collection. JHM, VM-S, JP-M, MC-B, RV-E, MM-S all contributed to analysis and writing of the paper.

Competing interests None declared.

Patient consent Obtained.

Provenance and peer review Not commissioned; externally peer reviewed.

(c) European Association of Hospital Pharmacists (unless otherwise stated in the text of the article) 2018. All rights reserved. No commercial use is permitted unless otherwise expressly granted.

\section{REFERENCES}

1 World Health Organization. Drugs for the elderly. Copenhagen: Regional Office for Europe, 1985.

2 Spinewine A, Schmader KE, Barber N, et al. Appropriate prescribing in elderly people: how well can it be measured and optimised? Lancet 2007:370:173-84.

3 Buitrago Ramírez F. Methods for measuring the suitability of pharmacological treatment in the elderly with multiple conditions and on multiple drugs. Aten Primaria 2013;45:19-20.

4 Delgado Silveira E, Fernandez Villalba EM, García-Mina Freire M, et al. Impacto de la intervención farmacéutica en el tratamiento del paciente mayor pluripatológico. Farm Hosp 2015:39:192-202.

5 Gavilán Moral E, Villafaina Barroso A. Concepto y factores determinantes de la polimedicación. Polimedicación Y Salud: estrategias para la adecuación terapéutica. Barcelona: Reprodisenny/Laboratorio de Prácticas Innovadoras en Polimedicación y Salud, 2011::23-33.

6 Gallagher P, Lang PO, Cherubini A, et al. Prevalence of potentially inappropriate prescribing in an acutely ill population of older patients admitted to six European hospitals. Eur J Clin Pharmacol 2011;67:1175-88.
7 Fialová D, Topinková E, Gambassi G, et al. Potentially inappropriate medication use among elderly home care patients in Europe. JAMA 2005;293:1348-58.

8 Carey IM, De Wilde S, Harris T, et al. What factors predict potentially inappropriate primary care prescribing in older people? Analysis of UK primary care patient record database. Drugs Aging 2008;25:693-706.

9 Gallagher P, Ryan C, Byrne S, et al. STOPP (screening tool of older person's prescriptions) and START (screening tool to alert doctors to right treatment). Consensus validation. Int J Clin Pharmacol Ther 2008;46:72-83.

10 Spinewine A, Swine C, Dhillon S, et al. Effect of a collaborative approach on the quality of prescribing for geriatric inpatients: a randomized, controlled trial. J Am Geriatr Soc 2007; 55:658-65.

11 Ollero Baturone M, Alvarez M, Baron-Franco B, et al. Proceso asistencial integrado. Sevilla: consejería De Salud, Junta de Andalucía. 2nd ed, 2007. http://www. juntadeandalucia.es/salud/sites/csalud/contenidos/Informacion_General/p_3_p_3_ procesos_asistenciales_integrados/pai/atencion_pacientes_pluripatologicos_v3? perfil=org. (accessed 22 Feb 2017).

12 Scott I, Jayathissa S. Quality of drug prescribing in older patients: is there a problem and can we improve it? Intern Med J 2010;40:7-18.

13 Ahmad A, Mast MR, Nijpels G, et al. Identification of drug-related problems of elderly patients discharged from hospital. Patient Prefer Adherence 2014;8:155-65.

14 Steinman MA, Hanlon JT. Managing medications in clinically complex elders: "There's got to be a happy medium". JAMA 2010;304:1592-601.

15 O'Sullivan D, O'Mahony D, O'Connor MN, et al. The impact of a structured pharmacist intervention on the appropriateness of prescribing in older hospitalized patients. Drugs Aging 2014;31:471-81.

16 Yeste-Gómez I, Durán-García ME, Muiño-Miguez A, et al. Potentially inappropriate prescriptions in the ambulatory treatment of elderly patients. Rev Calid Asist 2014;29:22-8.

17 Gallagher P, O'Mahony D. STOPP (Screening Tool of Older Persons' potentially inappropriate prescriptions): application to acutely ill elderly patients and comparison with Beers' criteria. Age Ageing 2008;37:673-9.

18 Gallagher PF, O'Connor MN, O'Mahony D. Prevention of potentially inappropriate prescribing for elderly patients: a randomized controlled trial using STOPP/START criteria. Clin Pharmacol Ther 2011:89:845-54.

19 San-José A, Agustí A, Vidal X, et al. Potentially inappropriate prescription in older patients in Spain (PIPOPS) Investigators' project. Inappropriate prescribing to the oldest old patients admitted to hospital: prevalence, most frequently used medicines, and associated factors. BMC Geriatr 2015;15:42.

20 Sevilla-Sánchez D, Espaulella-Panicot J, de Andrés-Lazaro AM, et al. [Potentially inappropriate medication on admission to a medium-stay unit according to STOPP and START criteria]. Rev Esp Geriatr Gerontol 2012;47:155-7.

21 Harlan M. Post-hospital syndrome-an acquired, transient condition of generalized risk N Engl J Med 2013:100-2.

22 O'Mahony D, O'Sullivan D, Byrne S, et al. STOPP/START criteria for potentially inappropriate prescribing in older people: version 2. Age Ageing 2015;44:213-8.

23 Wilson M, Mair A, Dreischulte T, et al. Prescribing to fit the needs of older people-the NHS Scotland Polypharmacy Guidance, 2nd edition. J R Coll Physicians Edinb 2015;45:108-13

24 Galvin R, Moriarty F, Cousins G, et al. Prevalence of potentially inappropriate prescribing and prescribing omissions in older Irish adults: findings from the Irish LongituDinal Study on ageing study (TILDA). Eur J Clin Pharmacol 2014;70:599-606. 